Gut and Liver, Vol. 14, No. 6, November 2020, pp. 826-832

\title{
Utility of Contrast-Enhanced Harmonic Endoscopic Ultrasound for the Guidance and Monitoring of Endoscopic Radiofrequency Ablation
}

\author{
Jun-Ho Choi ${ }^{1}$, Dong-Wan Seo ${ }^{2}$, Tae Jun Song ${ }^{2}$, Do Hyun Park ${ }^{2}$, Sang Soo Lee ${ }^{2}$, Sung Koo Lee ${ }^{2}$, and Myung-Hwan Kim ${ }^{2}$ \\ ${ }^{1}$ Department of Internal Medicine, Dankook University Hospital, Dankook University College of Medicine, Cheonan, and ${ }^{2}$ Department of \\ Internal Medicine, Asan Medical Center, University of Ulsan College of Medicine, Seoul, Korea
}

Background/Aims: Interventional endoscopists may utilize contrast-enhanced harmonic endoscopic ultrasound (CEHEUS) for image guidance during radiofrequency ablation (RFA) because of its capability to delineate real-time tumor perfusion dynamics. The purpose of this study was to assess the utility of CEH-EUS for the guidance and monitoring of endoscopic RFA. Methods: Nineteen consecutive patients with solid abdominal tumors who underwent CEH-EUS and endoscopic RFA were included. The extent of the ablation was assessed by CEH-EUS at 5 to 7 days after RFA. Additional RFAs were performed under CEH-EUS guidance. Results: The diagnoses were as follows: nonfunctioning neuroendocrine tumor $(n=13)$, solid pseudopapillary neoplasm (SPN) $(n=2)$, insulinoma $(n=1)$, left adrenal adenoma $(n=2)$, and left adrenal oligometastasis $(n=1)$. Pre-CEH-EUS findings revealed that 17 cases showed hyperenhanced patterns and two cases of SPN showed isoenhanced patterns. CEH-EUS-assisted RFA was technically feasible in all 19 patients. After the first RFA session, seven patients of the treated tumors showed the disappearance of intratumoral enhancement on CEH-EUS, whereas 12 showed residual contrast enhancement. Twelve patients with incomplete ablation were further treated with additional RFA under real-time CEH-EUS guidance. Radiologic complete response was observed in 13 patients (68.4\%). Among the 35 ablation procedures, the only adverse events were two episodes of pancreatitis (5.7\%; 1 moderate and 1 mild). During the median follow-up of 28 months, the local recurrence rate was $7.7 \%$. Conclusions: The application of CEH-EUS for RFA could be helpful in assessing early treatment response after ablation and targeting residual viable tumors during additional ablation sessions. (Gut Liver 2020;14:826-832)
Key Words: Contrast agent; Endosonography; Radiofrequency ablation

\section{INTRODUCTION}

With the advent of ablation technologies, endoscopic ultrasound (EUS)-guided ablation has emerged as a viable treatment option and an alternative to surgery in managing solid pancreatic tumors. There are variations of ablative procedures, including radiofrequency ablation (RFA), ethanol injection, and photodynamic therapy. ${ }^{1-4}$ These procedures involve the placement of the ablative probe into the target lesions under EUS guidance. ${ }^{5}$ Precise intra-procedure delineation of target lesions and post-procedure assessment of the ablation area are of utmost importance for effective treatment. Conventionally, procedural guidance can be achieved with EUS, ${ }^{6}$ which allows visualization of a needle while performing a procedure in a real-time manner. However, some lesions are poorly defined on gray-scale EUS, and there can be some difficulty differentiating ablative margin from necrotic tumor.

Contrast-enhanced harmonic EUS (CEH-EUS) is a safe and an easily available imaging modality. ${ }^{7,8}$ It has been shown to accurately depict tumor vascularity. ${ }^{8-10}$ Several studies have assessed the usefulness of this technique as a diagnostic tool in patients with pancreatic tumors. ${ }^{8,9,11}$ Contrast harmonic imaging can improve the detectability and localization of enhancing pancreatic tumors if the lesions cannot be adequately characterized on Bmode sonography. ${ }^{9}$ A newer application may be using CEH-EUS to guide ablation therapy for solid abdominal tumors because of its ability to delineate real-time tumor perfusion dynamics. ${ }^{12}$ However, the available data regarding CEH-EUS for tumor ablation are extremely limited. Therefore, the study aimed to evaluate the clinical utility of CEH-EUS-assisted RFA for solid abdominal tumors.

Correspondence to: Dong-Wan Seo

Division of Gastroenterology, Department of Internal Medicine, Asan Medical Center, University of Ulsan College of Medicine, 88 Olympic-ro 43gil, Songpa-gu, Seoul 05505, Korea

Tel: +82-2-3010-3192, Fax: +82-2-485-5782, E-mail: dwseoamc@amc.seoul.kr

Received on April 14, 2019. Revised on October 15, 2019. Accepted on October 23, 2019. Published online February 3, 2020.

pISSN 1976-2283 eISSN 2005-1212 https://doi.org/10.5009/gnl19123

@ This is an Open Access article distributed under the terms of the Creative Commons Attribution Non-Commercial License (http://creativecommons.org/licenses/by-nc/4.0) which permits unrestricted non-commercial use, distribution, and reproduction in any medium, provided the original work is properly cited. 


\section{METERIALS AND METHODS}

\section{Patients}

Between October 2014 and December 2017, 19 patients with solid abdominal tumors $3 \mathrm{~cm}$ or smaller who underwent CEHEUS assisted RFA were included in this study. The inclusion criteria were as follows: (1) the presence of a pancreatic neuroendocrine tumor (NET) or solid pseudopapillary neoplasm (SPN) $<3 \mathrm{~cm}$ with histological proof; (2) or a biochemically proven functioning adrenal adenoma, including a biopsy proven left adrenal oligo metastasis; and (3) patients with inoperability or refusal of surgery. A group of 10 patients reported in a previously published study was included in the present study. ${ }^{1}$ The exclusion criteria included: advanced lung or heart disease precluding adequate sedation, presence of a pacemaker, irreversible coagulopathy, and refusal to participate the study. Nonfunctioning NET, SPN, and adrenal metastasis were diagnosed using the histopathological results. The diagnosis of functioning NET was made based on the histopathological results, elevated hormone levels, and the presence of associated symptoms. The diagnosis of functioning adrenal adenoma was based on clinical and biochemical criteria.

The study was approved by the Institutional Review Board at Asan Medical Center (IRB number: 2016-0108), and written informed consent was obtained from all patients at the time of enrollment. The data were prospectively collected and retrospectively analyzed in this study.

\section{Methods}

At baseline, conventional EUS, CEH-EUS and contrastenhanced computed tomography (CT) for a complete evaluation of the target mass to characterize and stage the lesion vascularization were performed. After RFA, all patients underwent CEH-EUS within 5 to 7 days from ablation to assess the early treatment response of obtained necrotic area (absence of enhancement areas in the context of the treated lesion). If a residual enhancing tumor was detected on the CEH-EUS, a further RFA session was directly performed under real-time CEH imaging guidance.

\section{1) Contrast-enhanced harmonic EUS}

CEH-EUS was performed using a linear array echoendoscope (Olympus GF-UCT240; Olympus Medical System, Tokyo, Japan), and the images were analyzed using an Aloka Prosound Alpha a-10 system (Aloka Co. Ltd., Tokyo, Japan).

Prior to intravenous injection of the ultrasound contrast agent, the target lesion was scanned by gray-scale imaging to determine the location, echogenicity, margin, and size of the index tumor. The contrast harmonic specific mode was adjusted after the plane showing the area of tumor has been viewed on gray-scale EUS. Thus, we could see both gray-scale and contrast harmonic images side by side on the monitor in a real-time manner. After successful RFA, ablated area did not show vascular enhancement during CEH-EUS because there was no viable microvessel. Thus, it was possible to evaluate the residual tumor after RFA with these differential enhancement characteristics. SonoVue (Bracco, Milan, Italy) was initially used in our case series from 2014 to 2015. From 2016 onward, Sonazoid (Daiichi Sankyo, Tokyo, Japan) was used because it was available thereafter. Intravenous bolus injection of $2.5 \mathrm{~mL}$ of SonoVue was followed by a saline flush. Sonazoid was used at a dose of 0.015 $\mathrm{mL} / \mathrm{kg}$ bolus, followed by a flush $10 \mathrm{~mL}$ of saline. The CEH-EUS examinations lasted for 2 minutes from the ultrasound contrast agent injection. The patterns of enhancement were evaluated after the blood pool phase of the perfusion image.

\section{2) Radiofrequency ablation}

All patients were sedated with a combination of intravenous meperidine, midazolam, and/or propofol. We used an endoscopic RFA system (STARmed Co., Seoul, Korea) consisting of an 18- or 19-gauge prototype needle electrode (EUSRA ${ }^{\mathrm{TM}}$ ), a radiofrequency generator, and an inner cooling system to avoid charring of the electrode surface. The needle electrode was covered with a sheath, except for the exposed tip of $10 \mathrm{~mm}$ with a sharp conical tip for energy delivery.

The RFA needle electrode was introduced into the mass, crossing a minimum volume of normal surrounding pancreatic parenchyma and avoiding major vessels or pancreatic duct. After confirming the location of the needle electrode, $50 \mathrm{~W}$ ablation power was delivered at each site. On pressing the foot pedal switch once, after a slight lag, echogenic bubbles progressively start appearing around the needle electrode, indicative of effective RFA at the site. The time duration of ablation is in relation to tissue impedance measured in real-time manner by the radiofrequency generator. Energy delivery was controlled by a foot pedal switch. The RFA was repeated until the hyperechoic zone around the electrode sufficiently covered the entire tumor. In larger tumors, the needle electrode can be relocated under EUS visualization to treat non-ablated area along the same needle pathway closer to the echoendoscope. Additional needle passes using the fanning technique can be made to further ablate different areas within the target lesion. Technical precautions included securing a minimum 5-mm safety distances between the RFA needle electrode and surrounding structures (main pancreatic duct and vessels).

\section{Main outcomes and definitions}

The primary endpoint was to assess the feasibility and safety of CEH-EUS-assisted RFA. Technical success was defined as accurate placement of the ablation probe into target tumor with coverage of the tumor by the ablation zone on immediate EUS images. The safety endpoints were major adverse events including acute pancreatitis, bleeding, perforation, and injury to surrounding structures: minor adverse events including abdominal 


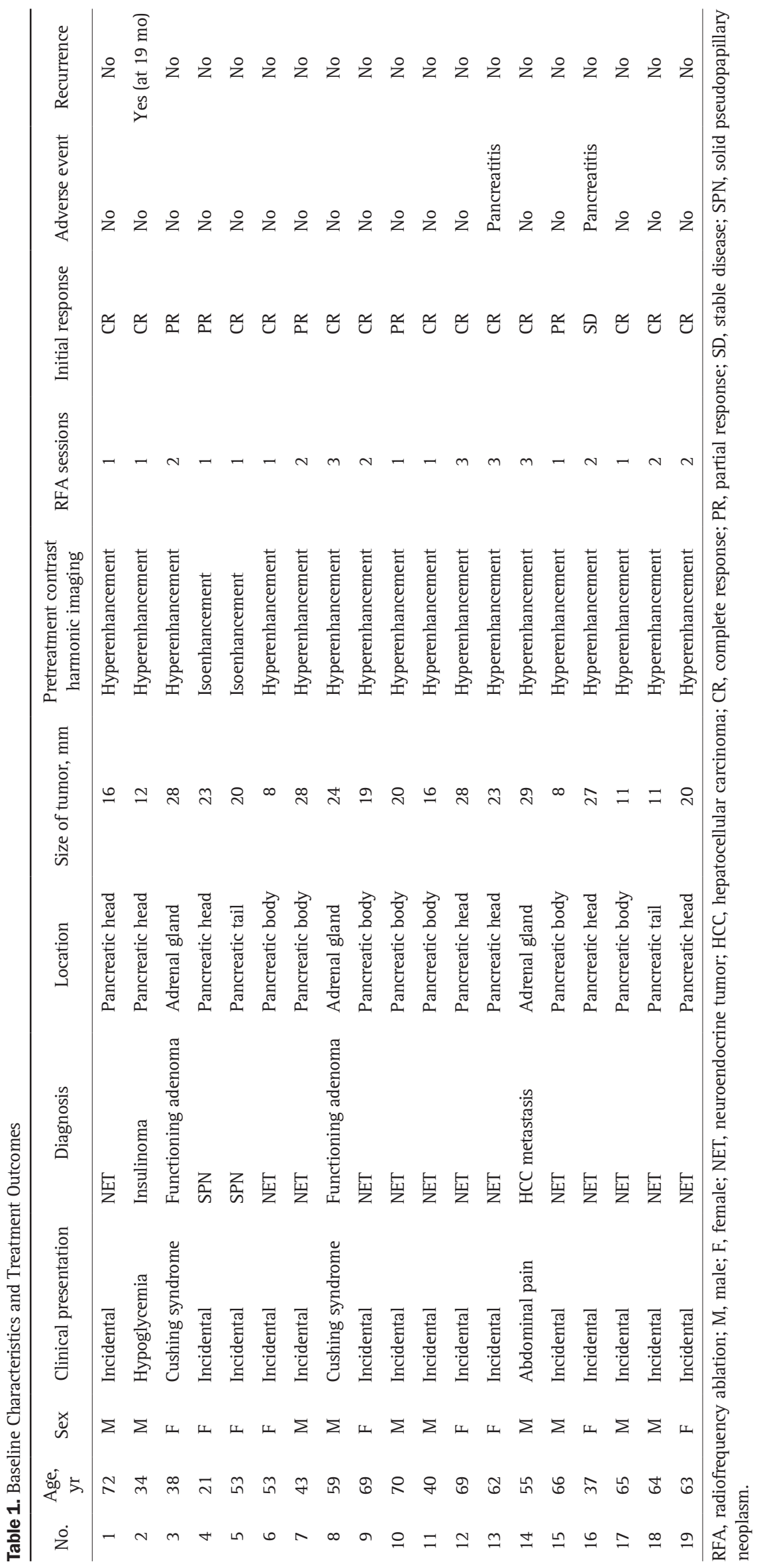


pain and fever. Adverse events were classified according to published criteria. The secondary endpoint was to assess the antitumor efficacy at 1 year follow-up, and the result was documented according to modified RECIST criteria ${ }^{13}$ (complete response [CR], disappearance of any intratumoral arterial enhancement in target lesions; partial response, at least a 30\% decrease in the sum of diameters of viable target lesions, taking as reference the baseline sum of the diameters of target lesions). Progressive disease and stable disease criteria were as follows: progressive disease-an increase of at least 20\% in the sum of the diameters of viable target lesions, taking as reference the smallest sum of the diameters of viable target lesions recorded since treatment started; stable disease-any cases that do not qualify for either partial response or progressive disease. These criteria were based on the serial change in enhancing patterns within the tumor on CEH-EUS and contrast-enhanced CT. Local recurrence was defined as a reappearance of an enhancing lesion and/or clinical symptom during the follow-up of a patient who had achieved a CR.

\section{Follow-up protocol}

The study patients were followed up at least 12 months after the ablation therapy. CEH-EUS was performed at 5 to 7 days after ablation to assess early treatment response. Imaging was repeated at 3 to 6 months and 1 year with CT and/or CEH-EUS.

\section{RESULTS}

Table 1 shows their baseline characteristics. The diagnoses were as follows: nonfunctioning NET ( $n=13)$, SPN ( $n=2)$, insulinoma ( $\mathrm{n}=1)$, Cushing syndrome due to left adrenal adenoma $(\mathrm{n}=2)$, and left adrenal oligometastasis of a hepatocellular carcinoma $(n=1)$. Of these 19 patients, 17 were pathologically confirmed by EUS-guided fine needle biopsy, and two patients with functioning adrenal adenoma were diagnosed based on biochemical and imaging findings. The median maximal diameter of the tumors was $20 \mathrm{~mm}$ (range, 8-29 mm). Pre-procedure CEH-EUS findings revealed that 17 cases showed hyperenhanced patterns and two cases of SPN had isoenhanced patterns (Table 1).

CEH-EUS-assisted RFA was technically feasible in all 19 patients; seven patients underwent one RFA session, eight patients
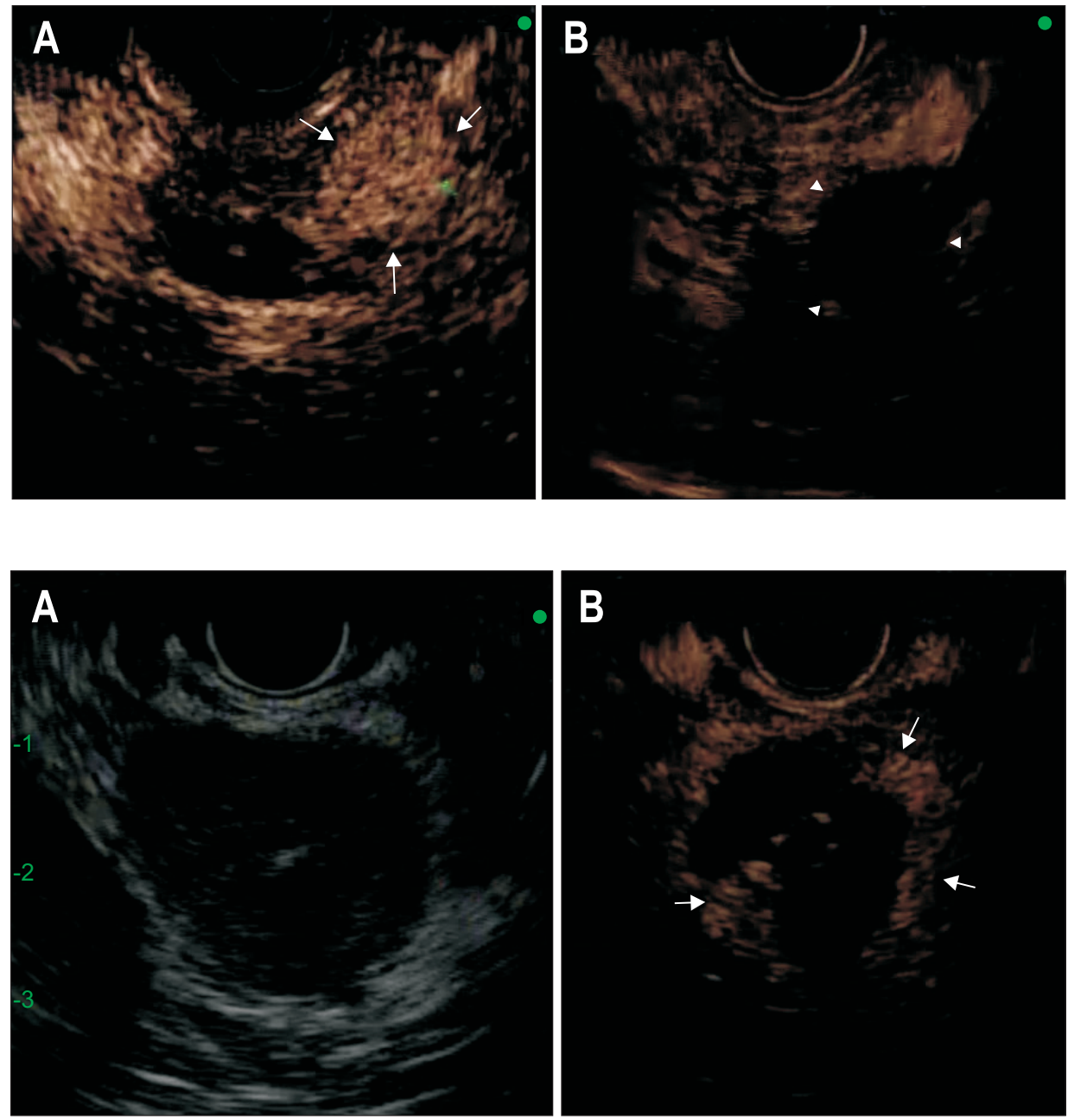

Fig. 1. A pre-CEH-EUS image shows homogeneous hyperenhancement (arrows) of the pancreatic neuroendocrine tumor (A). Five days after radiofrequency ablation, no enhancement (arrowheads) in the arterial phase is seen inside the tumor, compatible with a complete response (B).

CEH-EUS, contrast-enhanced harmonic endoscopic ultrasound.

Fig. 2. Conventional gray scale (A) and contrast-specific (ExPHD)-mode (B) after the first session of radiofrequency ablation. The B-mode EUS image (A) shows the adrenal adenoma as a hypoechoic area. The CEH-EUS image clearly shows an enhancing viable tumor for additional ablation (arrows).

CEH-EUS, contrast-enhanced harmonic endoscopic ultrasound. 
underwent two sessions, and four patients were managed with three sessions. The median number of RFA sessions was 2 (range, 1-3). After the first RFA session, seven of the ablated tumors showed no intratumoral enhancement on CEH-EUS (Fig. 1), whereas 12 showed peripheral nodular enhancement with nonenhanced areas in the center of the ablation zone. These 12 tumors were found to have residual enhancing foci indicative of a viable tumor (Fig. 2). Twelve patients with incomplete response were treated with additional RFAs under real-time CEH-EUS guidance. During the additional ablation sessions, CEH-EUS facilitated the accurate targeting of the RFA needle into the lesion to be treated (Fig. 3). A radiologic CR was achieved at 1 year in 13 of 19 tumors (68.4\%) (Fig. 4). Partial response was observed in five patients (26.3\%), and stable disease in one patient (5.3\%). The one patient with insulinoma experienced symptomatic relief from hypoglycemia and biochemical improvement. In two patients diagnosed with Cushing syndrome due to adrenal adenoma, serum and urine cortisol levels returned to normal following day. However, after the fourth month, the cortisol levels were re-elevated in one case, and the patient proceeded to surgery at this time.

During the follow-up (median, 28 months; range, 12 to 48 months) of the 13 patients with initial radiologic $\mathrm{CR}$, the tumor recurred in one (7.7\%) patient with NET within 19 months. No malignancy was discovered during follow-up.

Of the 35 ablation procedures, the adverse events included two episodes of pancreatitis $(5.7 \% ; 1$ moderate and 1 mild). Both patients with pancreatitis responded well to conservative treatment, requiring administration of intravenous fluids. In one patient who developed post-procedure pancreatitis, we inserted the prophylactic pancreatic duct stenting to prevent pancreatitis prior to 2nd session of RFA.

\section{DISCUSSION}

CEH-EUS-assisted RFA in patients with solid abdominal tumors is a feasible method with acceptable complication rate. Among 19 patients who underwent CEH-EUS-assisted RFA, $68.4 \%$ of the participants achieved a radiologic CR at 1 year. During the follow-up (median, 28 months; range, 12 to 48
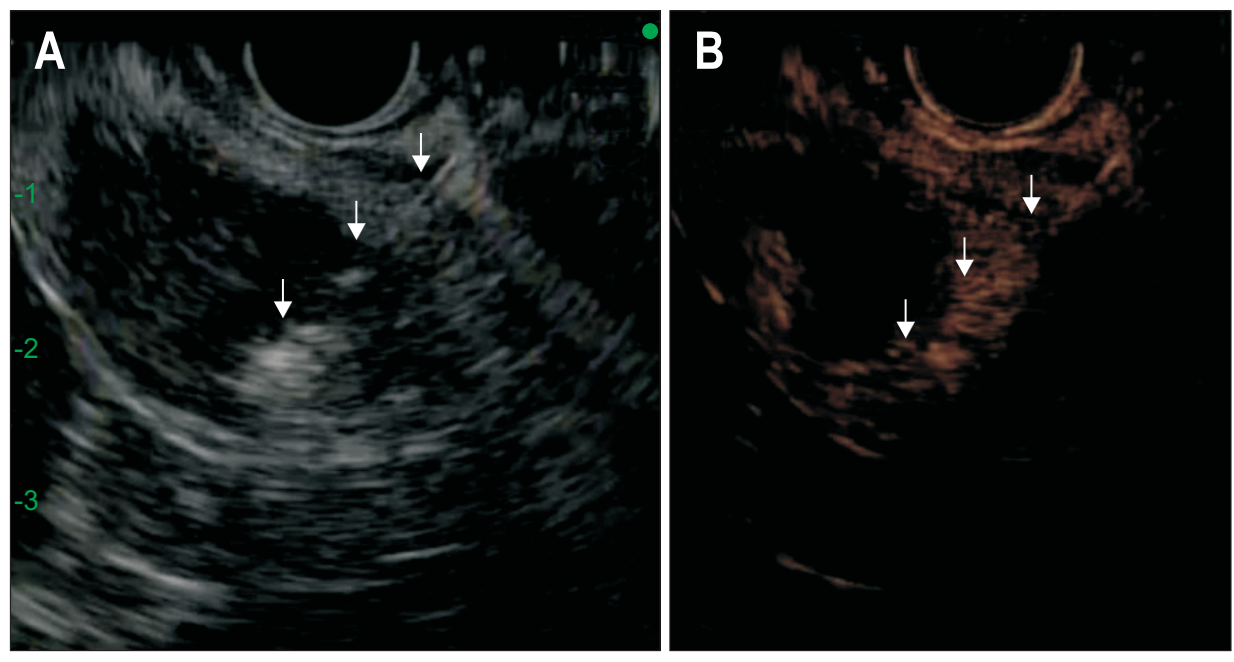

Fig. 3. Conventional gray scale (A) and contrast-specific (ExPHD)-mode (B) during the second session of radiofrequency ablation. An arterial phase CEH-EUS image (B) obtained 5 days after RFA shows peripheral eccentric enhancement, suggesting a viable tumor. CEH-EUS facilitates the accurate targeting of the RFA needle (arrows) into the lesion to be treated.

CEH-EUS, contrast-enhanced harmonic endoscopic ultrasound; RFA, radiofrequency ablation.
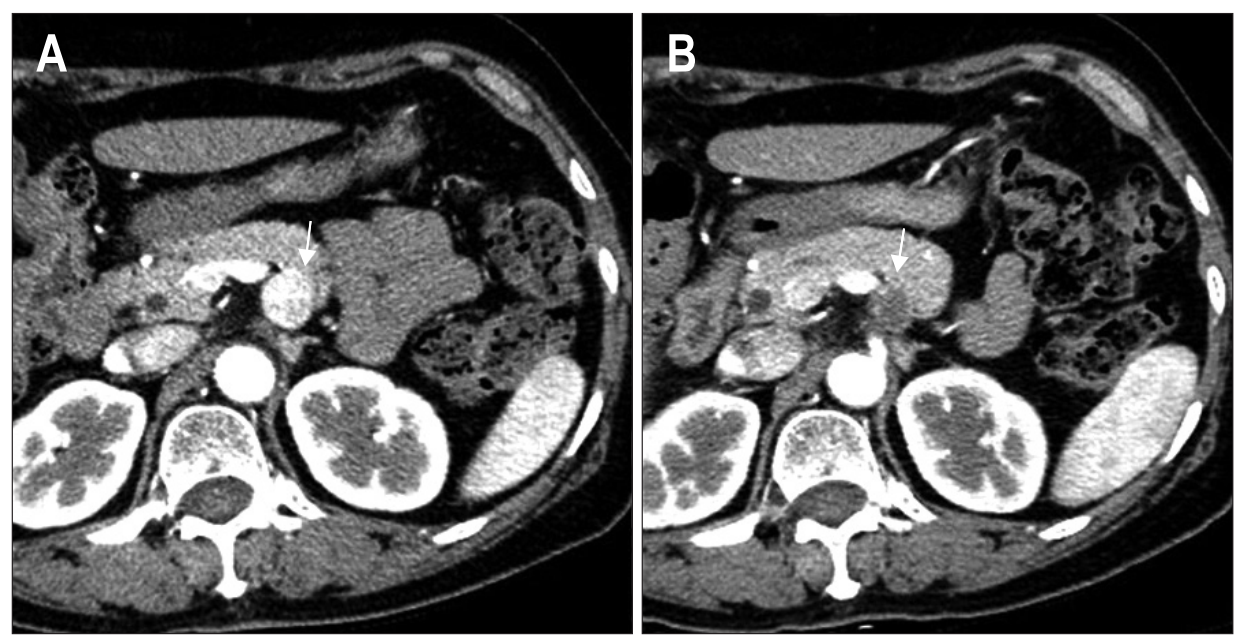

Fig. 4. (A) Abdominal computed tomography (CT) image obtained before ablation shows a $1.9-\mathrm{cm}$ neuroendocrine tumor in the body of the pancreas (arrow). (B) The axial arterial phase CT image from the 3-month follow-up depicting a no enhancing hypodense lesion (arrow). 
months) of the 13 patients who achieved initial CR, local recurrence was found in one patient (7.7 \%). The application of CEHEUS could be helpful in assessing early therapeutic responses and targeting the residual viable lesions during additional RFA sessions.

The advantages of CEH-EUS-assisted tumor ablation are: identifying remnant tumors, enabling assessment of early treatment responses, and guiding an accurate puncture for additional ablation therapy. On gray-scale EUS, the ablation zone generally appeared hypoechoic or mixed echogenic, and this method is unable to differentiate between viable and necrotic tissue inside the tumor. CEH-EUS can assess the effective ablation area by depicting the boundary of the nonenhanced zone. In this study, after the first session of RFA, seven of the treated tumors showed no intratumoral enhancement on CEH-EUS, whereas 12 revealed marginal nodular enhancement on CEHEUS. The usual appearance of a residual viable tumor was an eccentric or nodular peripheral enhancement on CEH-EUS. In 12 patients with residual tumors, CEH-EUS facilitated the accurate targeting of the RFA needle into the lesion to be treated. By directing the ablation needle electrode toward the contrastenhanced areas within the target lesion, effective ablation for the remnant tumor can be performed, potentially resulting in an increase in treatment efficacy.

Limited data are currently available for EUS-RFA in mainly small (less than $2 \mathrm{~cm}$ in diameter) pancreatic NETs from small case series including a total of seven patients. ${ }^{14-17}$ The outcomes reported complete ablation in two patients, central necrosis in one patient, and reduction of tumor size in three patients. ${ }^{14-17} \mathrm{~A}$ recent case series including only three insulinoma showed quick symptomatic and biochemical improvement within 2 days following the RFA, with sustained results at 2 years of follow-up. ${ }^{15}$ In the present study, the final therapeutic efficacy reached a CR in $63.1 \%$ of the tumors after median follow-up of 28 months. The outcomes in the present study seems to be close to those previously reported. Despite the use of the CEH-EUS, there are several reasons why treatment efficacy may appear to be lower than expected: first, in this study, 57.8\% of tumors (11/19) were more than $2 \mathrm{~cm}$ in diameter. In those cases, adequate tissue ablation is often difficult due to the potential risk of thermal injury, which increases the possibility of incomplete ablation and local recurrence. Secondly, to avoid an overestimation of therapeutic response, we adopted more strict criteria for the interpretation of a CR on a careful analysis of lesion enhancement in both CEH-EUS and CT. With the limitations of heterogeneity considered, more research on RFA techniques, design method, and the use of contrast agents might be needed to clarify the role of CEH-EUS.

CEH-EUS is increasingly used, the advantages over CT scan being the lack of radiation and real-time guidance and a high sensitivity in the detection of vascularization, even in small tumors. ${ }^{8}$ Endosonographers should be aware of the presence of confounding gas bubbles or post-ablation hyperemia at the ablation zone, which could make the assessment of CEH-EUS images confusing. ${ }^{18}$ Immediately after ablation, hyperemia is provoked around the ablation zone from tissue damage and the resultant inflammatory response. This inflammatory reaction often demonstrates a uniform rim of enhancement which, unlike residual viable tumors, persists throughout the different enhancement phases. Therefore, post-procedure CEH-EUS is recommended to be performed after a wait of at least 5 to 7 days.

A reasonable protocol in the follow-up of patients after RFA implies the use of CEH-EUS at about a week to detect residual tumors, with CT or magnetic resonance imaging being used at 3 to 6 months to detect marginal recurrence. In long-term followup, CEH-EUS and/or contrast-enhanced CT may be the mainstays for imaging of treated patients and the detection of local or remote relapse.

A limitation of our study is that we had no pathological proof of the ablated lesions. However, the use of a needle biopsy also had some limitations in proving residual tumors after ablation because of sampling errors. Therefore, most investigators relied on long-term follow-up to assess the therapeutic response by imaging technique. The present study was not a randomized controlled trial directly comparing CEH-EUS and conventional EUS. This study design was a compromise with given the fact that it was unethical not to use CEH-EUS.

In conclusion, CEH-EUS-assisted RFA might be a feasible and relatively safe method for management of solid abdominal tumors. The application of CEH-EUS for RFA may help to identify early treatment failures and can allow for accurate targeting for RFA. As the data accumulates, CEH-EUS may have the potential to reduce the number of surveillance CT performed, with the benefit of mitigating the need for nephrotoxic contrast agents. Further long-term follow-up studies with histologic evidence are necessary to confirm our conclusions.

\section{CONFLICTS OF INTEREST}

No potential conflict of interest relevant to this article was reported.

\section{ACKNOWLEDGEMENTS}

This study was supported by a grant from the Korean Health Technology R\&D project, Ministry of Health \& Welfare, Republic of Korea (grant number: HI16C1163).

\section{AUTHOR CONTRIBUTIONS}

Study concept and design: D.W.S. Data acquisition: J.H.C., D.W.S. Data analysis and interpretation: J.H.C. Drafting of the manuscript; critical revision of the manuscript for important intellectual content: J.H.C., D.W.S. Statistical analysis: J.H.C. Ad- 
ministrative, technical, or material support; study supervision: T.J.S., D.H.P., S.S.L., S.K.L., M.H.K., D.W.S. Approval of final manuscript: all authors.

\section{ORCID}

\section{Jun-Ho Choi \\ Dong-Wan Seo \\ Tae Jun Song \\ Do Hyun Park \\ Sang Soo Lee \\ Sung Koo Lee \\ Myung-Hwan Kim}

\section{REFERENCES}

1. Choi JH, Seo DW, Song TJ, et al. Endoscopic ultrasound-guided radiofrequency ablation for management of benign solid pancreatic tumors. Endoscopy 2018;50:1099-1104.

2. Park DH, Choi JH, Oh D, et al. Endoscopic ultrasonography-guided ethanol ablation for small pancreatic neuroendocrine tumors: results of a pilot study. Clin Endosc 2015;48:158-164.

3. Choi JH, Seo DW, Song TJ, et al. Long-term outcomes after endoscopic ultrasound-guided ablation of pancreatic cysts. Endoscopy 2017;49:866-873.

4. Choi JH, Oh D, Lee JH, et al. Initial human experience of endoscopic ultrasound-guided photodynamic therapy with a novel photosensitizer and a flexible laser-light catheter. Endoscopy 2015;47:1035-1038.

5. Lakhtakia S, Seo DW. Endoscopic ultrasonography-guided tumor ablation. Dig Endosc 2017;29:486-494.

6. Wallace MB, Sabbagh LC; EUS 2008 Working Group. EUS 2008 Working Group document: evaluation of EUS-guided tumor ablation. Gastrointest Endosc 2009;69(2 Suppl):S59-S63.

7. Choi JH, Seo DW. The expanding role of contrast-enhanced endoscopic ultrasound in pancreatobiliary disease. Gut Liver 2015;9:707-713.
8. Kitano M, Yamashita Y. New imaging techniques for endoscopic ultrasonography: contrast-enhanced endoscopic ultrasonography. Gastrointest Endosc Clin N Am 2017;27:569-583.

9. Fusaroli P, Napoleon B, Gincul R, et al. The clinical impact of ultrasound contrast agents in EUS: a systematic review according to the levels of evidence. Gastrointest Endosc 2016;84:587-596.

10. Lee TY, Cheon YK, Shim CS. Clinical role of contrast-enhanced harmonic endoscopic ultrasound in differentiating solid lesions of the pancreas: a single-center experience in Korea. Gut Liver 2013;7:599-604.

11. Kitano M, Kudo M, Yamao K, et al. Characterization of small solid tumors in the pancreas: the value of contrast-enhanced harmonic endoscopic ultrasonography. Am J Gastroenterol 2012;107:303310 .

12. Choi JH, Seo DW. Applications of contrast-enhanced harmonic endoscopic ultrasound on biliary, focal liver lesions and vascular diseases. Endosc Ultrasound 2017;6:21-24.

13. Lencioni R, Llovet JM. Modified RECIST (mRECIST) assessment for hepatocellular carcinoma. Semin Liver Dis 2010;30:52-60.

14. Pai M, Habib N, Senturk H, et al. Endoscopic ultrasound guided radiofrequency ablation, for pancreatic cystic neoplasms and neuroendocrine tumors. World J Gastrointest Surg 2015;7:52-59.

15. Lakhtakia S, Ramchandani M, Galasso D, et al. EUS-guided radiofrequency ablation for management of pancreatic insulinoma by using a novel needle electrode (with videos). Gastrointest Endosc 2016;83:234-239.

16. Waung JA, Todd JF, Keane MG, Pereira SP. Successful management of a sporadic pancreatic insulinoma by endoscopic ultrasound-guided radiofrequency ablation. Endoscopy 2016;48 Suppl 1:E144-E145.

17. Armellini E, Crinò SF, Ballarè M, Occhipinti P. Endoscopic ultrasound-guided radiofrequency ablation of a pancreatic neuroendocrine tumor. Endoscopy 2015;47 Suppl 1 UCTN:E600-E601.

18. Huang DY, Yusuf GT, Daneshi M, et al. Contrast-enhanced ultrasound (CEUS) in abdominal intervention. Abdom Radiol (NY) 2018;43:960-976. 